\title{
STRICT SOLUTION FOR A SECOND ORDER DIFFERENTIAL EQUATION IN HOLDER SPACES
}

\section{YOUCEF NAAS AND FATIMA ZOHRA MEZEGHRANI*}

Laboratory of Mathematics and Its Applications (LAMAP), University of Oran1, Oran 31000, Algeria

*Corresponding author: mezeghrani66@yahoo.fr

\begin{abstract}
In this paper we give some results on abstract second order differential elliptic equations of mixed type. The study is performed in Holder continuous Banach spaces. Our main purpose is the study of necessary and sufficient conditions on the data for obtaining existence, uniqueness and maximal regularity properties of the strict solution. The techniques used are based on analytic semigroups theory.
\end{abstract}

\section{INTRODUCTION}

. Let $X$ be a complex Banach space and consider the second order abstract differential problem

$$
u^{\prime \prime}(x)+A u(x)=f(x), x \in[0,1]
$$

with the boundary conditions

$$
u(0)=u_{0}, u^{\prime}(1)=u^{\prime}(0)
$$

Here $u_{0}$ is a given element in $X$ and $A$ is a closed linear operator of domain $D(A)$ not necessarily dense in $X$. We assume throughout the paper, the following ellipticity hypothesis:

$$
\forall \lambda \geqslant 0, \exists(A-\lambda)^{-1} \in \mathcal{L}(X):\left\|(A-\lambda I)^{-1}\right\|_{\mathcal{L}(X)} \leqslant \frac{c}{1+\lambda}
$$

Received 2019-05-23; accepted 2019-07-15; published 2019-11-01.

2010 Mathematics Subject Classification. 34G10, 34K10, 35J25, 47D03.

Key words and phrases. Abstract differential equation, boundary condition, analytic semigroup, Holder spaces, square root of operator, strict solution.

(C)2019 Authors retain the copyrights of their papers, and all open access articles are distributed under the terms of the Creative Commons Attribution License. 
Our study will treat the existence, uniqueness and regularity of the solution under assumption (1.3). Here $f$ belongs to $C^{\alpha}([0,1] ; X), 0<\alpha<1$. Let us recall, for the reader's convenience, that.

$$
C^{\alpha}([0,1] ; X)=\left\{f \in C([0,1] ; X) / \sup _{s, t \in[0,1] / t-s \neq 0} \frac{\| f(t)-f(s)||_{X}}{|t-s|^{\alpha}}<+\infty\right\}
$$

The main result in this work affirms that, under assumption (1.3), problem (1.1)-(1.2) has a unique strict solution, i.e a function $u$ such that:

$$
\begin{gathered}
u \in C^{2}([0,1] ; X) \cap C([0,1] ; D(A)) \\
\quad \text { if and only if } \\
u_{0} \in D(A) \text { and } A u_{0}-f(0) \in \overline{D(A)}
\end{gathered}
$$

and that:

$$
\begin{gathered}
u^{\prime \prime}, A u \in C^{\alpha}([0.1] ; X) \\
\text { if and only if } \\
u_{0} \in D(A) \text { and } A u_{0}-f(0) \in(D(A), X)_{1-\frac{\alpha}{2},+\infty}
\end{gathered}
$$

where for all $\alpha \in] 0,1[$,

$$
(D(A), X)_{1-\frac{\alpha}{2},+\infty}=\left\{x \in X / \sup _{t>0}\left\|t^{\frac{\alpha}{2}} A e^{A t} x\right\|_{X}<+\infty\right\}
$$

we call property (1.4) the maximal regularity of the solution. We give, then, an explicit representation of the solution using the square root of $-A$ and Krein's method [9] based on order reduction of the equation. All the components of the solution have been analyzed using the Sinestrari method [14], the Lion's reiteration theorem [10] and the semigroup techniques, see Krein [9]. Since the density of $D(A)$ is not assumed, we must carefully use the square root of $A$, see Martinez-Sanz [13]. Several authors have been interested by equation (1.1) as an abstract problem of elliptic type, i.e under assumption (1.3) with different boundary conditions, where the second member $f$ belongs to two classes of Banach spaces, $L^{p}$ and $C^{\alpha}([0,1] ; X)$. Different methods have been used; semigroups or Dunford integrals. We cite, at first, Da Prato and Grisvard theory on the sum of operators see [5]. In arendt [1] the author proved that problem:

$$
u^{\prime \prime}(x)+B(x) u^{\prime}(x)+A(x) u(x)=f(x), x \in(0, \delta)
$$

with

$$
u(0)=x, u^{\prime}(0)=y,
$$

has a unique solution $u$ such that $u \in W^{2, p}(0, \delta ; X) \cap L^{p}(0, \delta ; D(A))$ and $u^{\prime} \in L^{p}(0, \delta ; D(B))$, in the case where $D(A)$ and $D(B)$ embed continuously and densely into $X$ and $f$ belong to $L^{p}(0, \delta ; X)$. A new approach 
based on the semigroup techniques and fractional powers of operators have been developped by Favini et al [6] concerning the complete equation :

$$
u^{\prime \prime}(x)+2 B(x) u^{\prime}(x)+A(x) u(x)=f(x),
$$

under Dirichlet boundary conditions. In Batty et al [3], the authors studied maximal regularity results for problem (1.5) with boundary conditions:

$$
u(0)=u_{0}, u^{\prime}(0)=u_{1} .
$$

Where $f$ belongs to $B_{p q}^{\alpha}(0, T ; X)$ and $\left(u_{0}, u_{1}\right) \in\left(X, D_{B}, D_{A}\right)_{B_{p q}^{\alpha}}^{\alpha}, 1 \leqslant p \leqslant \infty$

$1 \leqslant q \leqslant \infty$ and $\alpha \in\left(0, \frac{1}{p}\right)$. In this paper, we are interested in the resolution of problem (1.1) with mixed boundary conditions (1.3) which make our study difficult especially when the domain of $A$ is not dense in $X$. We prove existence, uniqueness and maximal regularity of the strict solution. We also give some applications. The plan of this paper is as follows. In section 2 and 3 we will recall some semigroup properties, and give some useful. Technical results to analyze the representation of the solution $u$. Section 4 is devoted to the existence, uniqueness and maximal regularity of the strict solution. Finaly, section 5 contains an application to a partial differential equation.

\section{Preliminaries And Technical Results}

Definition 2.1. we say that $\left(e^{x Q}\right)_{x \geq 0}$ is a generalized analytic semigroup if $Q$ is a linear operator in $X$, with not dense domain and verifying:

$$
\left\{\begin{array}{l}
\rho(Q) \supset S_{\omega, \delta}=\left\{\lambda \in \mathbb{C} \backslash\{\omega\} /|\arg (\lambda-\omega)|<\frac{\pi}{2}+\delta\right\} \text { and } \\
\sup _{\lambda \in S_{\omega, \delta}}\left\|(\lambda-\omega)(\lambda I-Q)^{-1}\right\|_{\mathcal{L}(X)}<+\infty,
\end{array}\right.
$$

where $\omega \in \mathbb{R}$ and $\delta \in] 0, \frac{\pi}{2}\left[\right.$. In this case $\left(e^{x Q}\right)_{x \geq 0}$ is not supposed strongly continuous semigroup (see $E$. Sinestrari [14], A. Lunardi [12]).

Remark 2.1. we fix $\left.r>0, \delta_{0} \in\right] 0, \delta\left[\right.$ then $\left(e^{x Q}\right)_{x \geq 0}$ is defined by

$$
e^{x Q}=\left\{\begin{array}{l}
\frac{1}{2 i \pi} \int_{\gamma} e^{\lambda x}(\lambda I-Q)^{-1} d \lambda \text { if } x>0 \\
I \text { if } x=0
\end{array}\right.
$$

where $\gamma$ is the sectoriel boundary curve of $S_{\omega, \delta_{0}} \backslash B(\omega, r)$ oriented positively. The following results are valable for all operator $Q$ infinitesimal generater of analytic generalized semi group.

Proposition 2.1. $\quad$ (1) Let $\varphi \in X$. Then the two following assertions are equivalent:

(a) $e^{\cdot Q} \varphi \in C([0,1] ; X)$.

(b) $\varphi \in \overline{D(Q)}$. 
(2) Let $\theta \in] 0,1\left[, g \in C^{\theta}([0,1] ; X), \varphi \in X\right.$. Set

$$
v(x, \varphi, g, Q)=e^{x Q} \varphi+\int_{0}^{x} e^{(x-s) Q} g(s) d s, x \in[0,1] .
$$

Then the two following assertions are equivalent :

(a) $v \in C^{1}([0,1] ; X) \cap C([0,1] ; D(Q))$.

(b) $\varphi \in D(Q)$ and $g(0)+Q \varphi \in \overline{D(Q)}$.

Considering the well known real interpolation space

$$
(D(Q), X)_{1-\theta, \infty}=(X, D(Q))_{\theta, \infty}
$$

Proof. (see H. Triebel [16] p. 25 et 76). We have also:

Theorem 2.1. (1) Let $\theta \in] 0,1[$. Then the two following assertions are equivalent.

(a) $e^{\cdot Q} \varphi \in C^{\theta}([0,1] ; X)$.

(b) $\varphi \in(D(Q), X)_{1-\theta, \infty}$.

(2) Let $\varphi \in X, \theta \in] 0,1\left[\right.$ and $g \in C^{\theta}([0,1] ; X)$. Set

$$
v(x)=\int_{0}^{x} e^{(x-s) Q}[g(s)-g(0)] d s, x \in[0,1],
$$

Then

$$
v \in C^{1, \theta}([0,1] ; X) \cap C^{\theta}([0,1] ; D(Q)) .
$$

(3) Let $g \in C([0,1] ; X)$ and $\varphi \in X$. Set

$$
w(x)=e^{x Q} \varphi+\int_{0}^{x} e^{(x-s) Q} g(s) d s, x \in[0,1],
$$

Then the two following assertions are equivalent.

(a) $w \in C^{1, \theta}([0,1] ; X) \cap C^{\theta}([0,1] ; D(Q))$.

(b) $g \in C^{\theta}([0,1] ; X), \varphi \in D(Q)$ and $g(0)+Q \varphi \in(D(Q), X)_{1-\theta, \infty}$.

(4) Let $g \in C^{\theta}([0,1] ; X)$. Then

$$
\left.Q \int_{0}^{1} e^{s Q}(g(s)-g(0)) d s \in(D(Q)), X\right)_{1-\theta, \infty} .
$$

Proof. Statement 2 is obtained by applying the Da Prato-Grisvard sum theory [5]. Statement 3 which improves Statement 2 is due to E. Sinestrari [14], see also G. Da Prato [4].

Let $g$ and $h$ be two given $X$ valued functions defined on $[0,1]$ and $\theta \in] 0,1[$. We write

$$
g \simeq_{\theta} h,
$$

if

$$
g-h \in C^{\theta}([0,1] ; X) .
$$


Proposition 2.2. Let $h \in C^{\theta}([0,1] ; X), \varphi \in D(Q)$ and set

$$
w(x)=e^{x Q} \varphi+\int_{0}^{x} e^{(x-s) Q} h(s) d s, x \in[0,1]
$$

Then

$$
Q w(\cdot) \simeq_{\theta} e^{\cdot Q}(Q \varphi+h(0))
$$

Proof. It is an easy consequence of Theorem 2.1 and Proposition 1.2, statement (ii) in Sinestrari [14].

Proposition 2.3. Assume (1.3). The operator $(I-Z)$ has a bounded inverse given by

$$
(I-Z)^{-1}=\frac{1}{2 \pi i} \int_{\gamma^{\#}} \frac{e^{2 z}}{I-e^{2 z}}(z I+B)^{-1} d z+I .
$$

where $\gamma^{\#}$ is a suitable curve in the complex plane.

Proof. see Lunardi [12].

We put throughout the paper

$$
B=\sqrt{-A} \text { and } Z=e^{-2 B}
$$

Remark 2.2. Hypothesis (1.3) implies that the operator $(-\sqrt{-A})$ generates an analytic semigroup denoted by $\left(e^{-\sqrt{-A} x}\right)_{x \geqslant 0}$ on $X$, see for instance Balakrishnan [2].

For $u_{0} \in X$, consider the follwing abstract function

$$
\begin{array}{ccc}
\left.\left.\mathcal{Q}_{0}:\right] 0,1\right] & \longrightarrow & X \\
x & \longmapsto & \mathcal{Q}_{0}(x, B) u_{0}
\end{array}
$$

defined by $\mathcal{Q}_{0}$

$$
\mathcal{Q}_{0}(x, B) u_{0}=(I-Z)^{-1}\left(I-e^{-B}\right)^{-2} e^{-x B} u_{0} .
$$

we have the following result

Lemma 2.1. we have:

(1) $\left.\left.\mathcal{Q}_{0}(\cdot, B) u_{0} \in C^{\infty}(] 0,1\right] ; D\left(A^{k}\right)\right), k \in \mathbb{N}$

(2) $\forall x \in] 0,1], \mathcal{Q}_{0}^{\prime \prime}(x, B) u_{0}+A \mathcal{Q}_{0}(x, B) u_{0}=0$,

(3) $\exists C>0, \forall x \in] 0,1],\left\|\mathcal{Q}_{0}(x, B) u_{0}\right\|_{X} \leq C\left\|u_{0}\right\|_{X}$.

Proof. (1) Let $x>0, u_{0} \in X$. It is not difficult to see that

$$
(I-Z)^{-1}\left(I-e^{-B}\right)^{-2} e^{-B x}=e^{-B x}(I-Z)^{-1}\left(I-e^{-B}\right)^{-2},
$$

therefore

$$
\mathcal{Q}_{0}(x, B) u_{0}=e^{-x B}(I-Z)^{-1}\left(I-e^{-B}\right)^{-2} u_{0},
$$

Hence we deduce the first statement using proposition 1.1 in Sinestrari [14]. 
(2) For $x \in] 0,1]$, we have:

$$
\begin{aligned}
\mathcal{Q}_{0}^{\prime}(x, B) u_{0} & =-(I-Z)^{-1}\left(I-e^{-B}\right)^{-2} e^{-x B} B u_{0} \\
& =-(I-Z)^{-1}\left(I-e^{-B}\right)^{-2} e^{-x B} \sqrt{A} u_{0}
\end{aligned}
$$

Therefore

$$
\begin{aligned}
\mathcal{Q}_{0}^{\prime \prime}(x, B) u_{0} & =+(I-Z)^{-1}\left(I-e^{-B}\right)^{-2} e^{-x B} B^{2} u_{0} \\
& =-(I-Z)^{-1}\left(I-e^{-B}\right)^{-2} e^{-x B} A u_{0}
\end{aligned}
$$

and

$$
\begin{aligned}
\mathcal{Q}_{0}^{\prime \prime}(x, B) u_{0}+A \mathcal{Q}_{0}(x, B) u_{0} & =-(I-Z)^{-1}\left(I-e^{-B}\right)^{-2} e^{-x B} A u_{0}-A(I-Z)^{-1}\left(I-e^{-B}\right)^{-2} e^{-x B} u_{0} \\
& =-(I-Z)^{-1}\left(I-e^{-B}\right)^{-2} e^{-x B} A u_{0}-(I-Z)^{-1}\left(I-e^{-B}\right)^{-2} e^{-x B} A u_{0} \\
& =0
\end{aligned}
$$

(3) It is well known that there exists $M>0$ such that for any $x>0, u_{0} \in X$

$$
\left\|e^{-B x} u_{0}\right\|_{X} \leq M\left\|u_{0}\right\|_{X}
$$

(see Tanabe [15], page 66, formula (3.27)). Thus, $\exists C>0$ :

$$
\begin{aligned}
\left\|\mathcal{Q}_{0}(x, B) u_{0}\right\|_{X} & =\left\|(I-Z)^{-1}\left(I-e^{-B}\right)^{-2} e^{-x B} u_{0}\right\|_{X} \\
& \leq C\left\|u_{0}\right\|_{X} .
\end{aligned}
$$

Let us specify the behavior of $\mathcal{Q}_{0}(., B)$ near 0 .

Lemma 2.2. (1) Let $u_{0} \in X$. Then

$$
\mathcal{Q}_{0}(\cdot, \sqrt{-A}) u_{0} \in C([0,1] ; X) \text { if and only if } u_{0} \in \overline{D(A)}
$$

(2) Let $u_{0} \in D(A)$. Then

$$
\mathcal{Q}_{0}(\cdot, \sqrt{-A}) u_{0} \in C([0,1] ; D(A)) \text { if and only if } A u_{0} \in \overline{D(A)}
$$

Proof. This result is a consequence of commutativity of $(I-Z)^{-1}\left(I-e^{-B}\right)^{-2}$ and $A$ on $D(A)$ ( see Sinestrari [14], Proposition 1.2, (ii), page 20), we also use the fact that

$$
\overline{D(\sqrt{-A})}=\overline{D(A)}
$$

see Haase [8], Corollary 3.1.11. Page 59. 
For $u_{0} \in X$, consider the following abstract function :

$$
\begin{array}{ccc}
\mathcal{Q}_{1}:[0,1[ & \longrightarrow & X \\
x & \longmapsto & \mathcal{Q}_{1}(x, B) u_{0}
\end{array}
$$

where

$$
\mathcal{Q}_{1}(x, B) u_{0}=-(I-Z)^{-1}\left(I-e^{-B}\right)^{-2} e^{-(1-x) B} u_{0}
$$

We have the following result :

Lemma 2.3. we have :

(1) $\mathcal{Q}_{1}(\cdot, \sqrt{-A}) u_{0} \in C^{\infty}\left(\left[0,1\left[; D\left(A^{k}\right)\right), k \in \mathbb{N}\right.\right.$

(2) $\forall x \in\left[0,1\left[, \mathcal{Q}_{1}^{\prime \prime}(x, \sqrt{-A}) u_{0}+A \mathcal{Q}_{1}(x, \sqrt{-A}) u_{0}=0\right.\right.$,

(3) $\exists C>0, \forall x \in\left[0,1\left[,\left\|\mathcal{Q}_{1}(x, \sqrt{-A}) u_{0}\right\|_{X} \leq C\left\|u_{0}\right\|_{X}\right.\right.$.

Proof. It is not difficult to prove this lemma, it is sufficient to replace $x$ by $1-x$.

Lemma 2.4. (1) Let $u_{0} \in X$. Then

$$
\mathcal{Q}_{1}(\cdot, \sqrt{-A}) u_{0} \in C([0,1] ; X) \text { if and only if } u_{0} \in \overline{D(A)}
$$

(2) Let $u_{0} \in D(A)$. Then

$$
\mathcal{Q}_{1}(\cdot, \sqrt{-A}) u_{0} \in C([0,1] ; D(A)) \text { if and only if } A u_{0} \in \overline{D(A)}
$$

Proof. The proof of this lemma is the same as lemma 2.2

\section{Representation of the Solution}

In this section we assume that (1.3) holds we set :

$$
u(1)=u_{1}
$$

Let us suppose that problem (1.1)-(1.2) has a strict solution $u$. Then $u$ is the strict solution of the following problem:

$$
\left\{\begin{array}{l}
u^{\prime \prime}(x)-B^{2} u(x)=f(x) \\
u(0)=u_{0} \\
u(1)=u_{1}
\end{array}\right.
$$

Therefore $u$ is represented by :

$$
u(x)=e^{-x B} \xi_{0}+e^{-(1-x) B} \xi_{1}-\frac{1}{2} B^{-1} \int_{0}^{x} e^{-(x-s) B} f(s) d s-\frac{1}{2} B^{-1} \int_{x}^{1} e^{-(s-x) B} f(s) d s
$$


where :

$$
\begin{gathered}
\xi_{0}=(I-Z)^{-1}\left(u_{0}-e^{-B} u_{1}\right)+\frac{1}{2}(I-Z)^{-1} B^{-1}\left(\int_{0}^{1} e^{-s B} f(s) d s-\int_{0}^{1} e^{-(2-s) B} f(s) d s\right) \\
\xi_{1}=(I-Z)^{-1}\left(-e^{-B} u_{0}+u_{1}\right)+\frac{1}{2}(I-Z)^{-1} B^{-1}\left(\int_{0}^{1} e^{-(1-s) B} f(s) d s-\int_{0}^{1} e^{-(1+s) B} f(s) d s\right)
\end{gathered}
$$

(see $[6]$ ). By derivation we obtain

$$
u^{\prime}(x)=-B e^{-x B} \xi_{0}+B e^{-(1-x) B} \xi_{1}+\frac{1}{2} \int_{0}^{x} e^{-(x-s) B} f(s) d s-\frac{1}{2} \int_{x}^{1} e^{-(s-x) B} f(s) d s .
$$

Using that $u^{\prime}(0)=u^{\prime}(1)$ we obtain

$$
-B \xi_{0}+B e^{-B} \xi_{1}-\frac{1}{2} \int_{0}^{1} e^{-s B} f(s) d s=-B e^{-B} \xi_{0}+B \xi_{1}+\frac{1}{2} \int_{0}^{1} e^{-(1-s) B} f(s) d s .
$$

Then we deduce :

$$
\begin{aligned}
u_{1}= & -u_{0}-B^{-1}\left(I-e^{-B}\right)^{-2} \int_{0}^{1} e^{-s B} f(s) d s+B^{-1}\left(I-e^{-B}\right)^{-2} \int_{0}^{1} e^{-(2-s) B} f(s) d s \\
& +B^{-1}\left(I-e^{-B}\right)^{-2} \int_{0}^{1} e^{-(1+s) B} f(s) d s-B^{-1}\left(I-e^{-B}\right)^{-2} \int_{0}^{1} e^{-(1-s) B} f(s) d s .
\end{aligned}
$$

Therefore $u$ is formally given by :

$$
\begin{aligned}
u(x)= & +(I-Z)^{-1}\left(I-e^{-B}\right)^{-2} e^{-x B} u_{0}-(I-Z)^{-1}\left(I-e^{-B}\right)^{-2} e^{-(1-x) B} u_{0} \\
& -(I-Z)^{-1}\left(I-e^{-B}\right)^{-2} e^{-(4-x) B} u_{0}-(I-Z)^{-1}\left(I-e^{-B}\right)^{-2} e^{-(2+x) B} u_{0} \\
& +(I-Z)^{-1}\left(I-e^{-B}\right)^{-2} e^{-(3+x) B} u_{0}-(I-Z)^{-1}\left(I-e^{-B}\right)^{-2} e^{-(1+x) B} u_{0} \\
& +(I-Z)^{-1}\left(I-e^{-B}\right)^{-2} e^{-(2-x) B} u_{0}+(I-Z)^{-1}\left(I-e^{-B}\right)^{-2} e^{-(3-x) B} u_{0} \\
& +\frac{1}{2}(I-Z)^{-1}\left(I-e^{-B}\right)^{-2} B^{-1} \int_{0}^{1} e^{-(x+s) B} f(s) d s \\
& -\frac{1}{2}(I-Z)^{-1}\left(I-e^{-B}\right)^{-2} B^{-1} \int_{0}^{1} e^{-(2+x+s) B} f(s) d s \\
& +\frac{1}{2}(I-Z)^{-1}\left(I-e^{-B}\right)^{-2} B^{-1} \int_{0}^{1} e^{-(2+x-s) B} f(s) d s \\
& -\frac{1}{2}(I-Z)^{-1}\left(I-e^{-B}\right)^{-2} B^{-1} \int_{0}^{1} e^{-(4+x-s) B} f(s) d s \\
& -(I-Z)^{-1}\left(I-e^{-B}\right)^{-2} B^{-1} \int_{0}^{1} e^{-(1-x+s) B} f(s) d s \\
& +(I-Z)^{-1}\left(I-e^{-B}\right)^{-2} B^{-1} \int_{0}^{1} e^{-(3-x+s) B} f(s) d s \\
& +\frac{1}{2}(I-Z)^{-1}\left(I-e^{-B}\right)^{-2} B^{-1} \int_{0}^{1} e^{-(2-x+s) B} f(s) d s \\
& -\frac{1}{2}(I-Z)^{-1}\left(I-e^{-B}\right)^{-2} B^{-1} \int_{0}^{1} e^{-(4-x+s) B} f(s) d s
\end{aligned}
$$




$$
\begin{aligned}
& -\frac{1}{2}(I-Z)^{-1}\left(I-e^{-B}\right)^{-2} B^{-1} \int_{0}^{1} e^{-(2-x-s) B} f(s) d s \\
& +\frac{1}{2}(I-Z)^{-1}\left(I-e^{-B}\right)^{-2} B^{-1} \int_{0}^{1} e^{-(4-x-s) B} f(s) d s \\
& -\frac{1}{2} B^{-1} \int_{0}^{x} e^{-(x-s) B} f(s) d s-\frac{1}{2} B^{-1} \int_{x}^{1} e^{-(s-x) B} f(s) d s
\end{aligned}
$$

\section{Existence, Uniqueness and Maximal Regularity}

Theorem 4.1. Let $f \in C^{\alpha}([0,1] ; X) ; 0<\alpha<1$, we assume that (1.3) holds. Then the following assertions are equivalent.

(1) problem (1.1)-(1.2) has a unique strict solution $u$, that is

$$
u \in C^{2}([0,1] ; X) \cap C([0,1] ; D(A))
$$

(i.e u satisfies (1.1)-(1.2))

(2)

$$
u_{0} \in D(A) \text { and } A u_{0}-f(0) \in \overline{D(A)}
$$

Proof. suppose that statement 2 holds, i.e

$$
u_{0} \in D(A) \text { and } A u_{0}-f(0) \in \overline{D(A)}
$$

The solution $u$ of problem (1.1)-(1.2) is given by (3.2), then

$$
\begin{aligned}
u^{\prime \prime}(x)= & -(I-Z)^{-1}\left(I-e^{-B}\right)^{-2} e^{-x B} A u_{0}+(I-Z)^{-1}\left(I-e^{-B}\right)^{-2} e^{-(1-x) B} A u_{0} \\
& +(I-Z)^{-1}\left(I-e^{-B}\right)^{-2} e^{-(4-x) B} A u_{0}+(I-Z)^{-1}\left(I-e^{-B}\right)^{-2} e^{-(2+x) B} A u_{0} \\
& -(I-Z)^{-1}\left(I-e^{-B}\right)^{-2} e^{-(3+x) B} A u_{0}+(I-Z)^{-1}\left(I-e^{-B}\right)^{-2} e^{-(1+x) B} A u_{0} \\
& -(I-Z)^{-1}\left(I-e^{-B}\right)^{-2} e^{-(2-x) B} A u_{0}-(I-Z)^{-1}\left(I-e^{-B}\right)^{-2} e^{-(3-x) B} A u_{0} \\
& +\frac{1}{2}(I-Z)^{-1}\left(I-e^{-B}\right)^{-2}\left(B \int_{0}^{1} e^{-(x+s) B} f(s) d s-B \int_{0}^{1} e^{-(2+x+s) B} f(s) d s\right) \\
& +\frac{1}{2}(I-Z)^{-1}\left(I-e^{-B}\right)^{-2}\left(B \int_{0}^{1} e^{-(2+x-s) B} f(s) d s-B \int_{0}^{1} e^{-(4+x-s) B} f(s) d s\right) \\
& +(I-Z)^{-1}\left(I-e^{-B}\right)^{-2}\left(B \int_{0}^{1} e^{-(3-x+s) B} f(s) d s-B \int_{0}^{1} e^{-(1-x+s) B} f(s) d s\right) \\
& +\frac{1}{2}(I-Z)^{-1}\left(I-e^{-B}\right)^{-2}\left(B \int_{0}^{1} e^{-(2-x+s) B} f(s) d s-B \int_{0}^{1} e^{-(4-x+s) B} f(s) d s\right) \\
& +\frac{1}{2}(I-Z)^{-1}\left(I-e^{-B}\right)^{-2}\left(B \int_{0}^{1} e^{-(4-x-s) B} f(s) d s-B \int_{0}^{1} e^{-(2-x-s) B} f(s) d s\right) \\
& -\frac{1}{2} B \int_{0}^{x} e^{-(x-s) B} f(s) d s-\frac{1}{2} B \int_{x}^{1} e^{-(s-x) B} f(s) d s \\
+ & f(x)
\end{aligned}
$$

We write $u^{\prime \prime}$ as 


$$
\begin{aligned}
& u^{\prime \prime}(x)=-(I-Z)^{-1}\left(I-e^{-B}\right)^{-2}\left(A u_{0}-f(0)\right) e^{-x B}+(I-Z)^{-1}\left(I-e^{-B}\right)^{-2}\left(A u_{0}-f(0)\right) e^{-(1-x) B} \\
& +(I-Z)^{-1}\left(I-e^{-B}\right)^{-2}\left(A u_{0}+f(1)\right) e^{-(4-x) B}+(I-Z)^{-1}\left(I-e^{-B}\right)^{-2} e^{-(2+x) B} A u_{0} \\
& -(I-Z)^{-1}\left(I-e^{-B}\right)^{-2}\left(A u_{0}-f(0)\right) e^{-(3+x) B}+(I-Z)^{-1}\left(I-e^{-B}\right)^{-2} e^{-(1+x) B} A u_{0} \\
& -(I-Z)^{-1}\left(I-e^{-B}\right)^{-2}\left(A u_{0}-f(0)\right) e^{-(2-x) B}-(I-Z)^{-1}\left(I-e^{-B}\right)^{-2} e^{-(3-x) B} A u_{0} \\
& +\frac{1}{2}(I-Z)^{-1}\left(I-e^{-B}\right)^{-2}\left(e^{-x B} B \int_{0}^{1} e^{-s B}(f(s)-f(0)) d s-B \int_{0}^{1} e^{-(2+x+s) B} f(s) d s\right) \\
& +\frac{1}{2}(I-Z)^{-1}\left(I-e^{-B}\right)^{-2}\left(B \int_{0}^{1} e^{-(2+x-s) B} f(s) d s-B \int_{0}^{1} e^{-(4+x-s) B} f(s) d s\right) \\
& +(I-Z)^{-1}\left(I-e^{-B}\right)^{-2}\left(B \int_{0}^{1} e^{-(3-x+s) B} f(s) d s-e^{-(1-x) B} B \int_{0}^{1} e^{-s B}(f(s)-f(0)) d s\right) \\
& +\frac{1}{2}(I-Z)^{-1}\left(I-e^{-B}\right)^{-2}\left(B \int_{0}^{1} e^{-(2-x+s) B} f(s) d s-B \int_{0}^{1} e^{-(4-x+s) B} f(s) d s\right) \\
& +\frac{1}{2}(I-Z)^{-1}\left(I-e^{-B}\right)^{-2} B\left(\int_{0}^{1} e^{-(4-x-s) B} f(s) d s-e^{-(1-x) B} \int_{0}^{1} e^{-(1-s) B}(f(s)-f(1)) d s\right) \\
& -\frac{1}{2}(I-Z)^{-1}\left(I-e^{-B}\right)^{-2} e^{-(2-x) B} f(1)-\frac{1}{2}(I-Z)^{-1}\left(I-e^{-B}\right)^{-2} e^{-(5-x) B} f(1) \\
& -\frac{1}{2}(I-Z)^{-1}\left(I-e^{-B}\right)^{-2} e^{-(4+x) B} f(0)-\frac{3}{2}(I-Z)^{-1}\left(I-e^{-B}\right)^{-2} e^{-(1+x) B} f(0) \\
& -\frac{1}{2} B \int_{0}^{x} e^{-(x-s) B}(f(s)-f(x)) d s-\frac{1}{2} B \int_{x}^{1} e^{-(s-x) B}(f(s)-f(x)) d s \\
& +\frac{1}{2}(f(x)-f(0)) e^{-x B}+\frac{1}{2}(f(x)-f(1)) e^{-(1-x) B}
\end{aligned}
$$

In view of lemmas 2.2 and 2.4 the first and second terms are in $C([0,1] ; X)$ and all other terms are continuous since $f \in C^{\alpha}([0,1] ; X)$. From which we deduce that $u^{\prime \prime}$ is in $C([0,1] ; X)$. By the same way, we write:

$$
\begin{aligned}
A u(x)= & +(I-Z)^{-1}\left(I-e^{-B}\right)^{-2} e^{-x B} A u_{0}-(I-Z)^{-1}\left(I-e^{-B}\right)^{-2} e^{-(1-x) B} A u_{0} \\
& -(I-Z)^{-1}\left(I-e^{-B}\right)^{-2} e^{-(4-x) B} A u_{0}-(I-Z)^{-1}\left(I-e^{-B}\right)^{-2} e^{-(2+x) B} A u_{0} \\
& +(I-Z)^{-1}\left(I-e^{-B}\right)^{-2} e^{-(3+x) B} A u_{0}-(I-Z)^{-1}\left(I-e^{-B}\right)^{-2} e^{-(1+x) B} A u_{0} \\
& +(I-Z)^{-1}\left(I-e^{-B}\right)^{-2} e^{-(2-x) B} A u_{0}+(I-Z)^{-1}\left(I-e^{-B}\right)^{-2} e^{-(3-x) B} A u_{0} \\
& -\frac{1}{2}(I-Z)^{-1}\left(I-e^{-B}\right)^{-2} B \int_{0}^{1} e^{-(x+s) B} f(s) d s \\
& +\frac{1}{2}(I-Z)^{-1}\left(I-e^{-B}\right)^{-2} B \int_{0}^{1} e^{-(2+x+s) B} f(s) d s \\
& -\frac{1}{2}(I-Z)^{-1}\left(I-e^{-B}\right)^{-2} B \int_{0}^{1} e^{-(2+x-s) B} f(s) d s \\
& +\frac{1}{2}(I-Z)^{-1}\left(I-e^{-B}\right)^{-2} B \int_{0}^{1} e^{-(4+x-s) B} f(s) d s \\
& +(I-Z)^{-1}\left(I-e^{-B}\right)^{-2} B \int_{0}^{1} e^{-(1-x+s) B} f(s) d s
\end{aligned}
$$




$$
\begin{aligned}
& -(I-Z)^{-1}\left(I-e^{-B}\right)^{-2} B \int_{0}^{1} e^{-(3-x+s) B} f(s) d s \\
& -\frac{1}{2}(I-Z)^{-1}\left(I-e^{-B}\right)^{-2} B \int_{0}^{1} e^{-(2-x+s) B} f(s) d s \\
& +\frac{1}{2}(I-Z)^{-1}\left(I-e^{-B}\right)^{-2} B \int_{0}^{1} e^{-(4-x+s) B} f(s) d s \\
& +\frac{1}{2}(I-Z)^{-1}\left(I-e^{-B}\right)^{-2} B \int_{0}^{1} e^{-(2-x-s) B} f(s) d s \\
& -\frac{1}{2}(I-Z)^{-1}\left(I-e^{-B}\right)^{-2} B \int_{0}^{1} e^{-(4-x-s) B} f(s) d s \\
& +\frac{1}{2} B \int_{0}^{x} e^{-(x-s) B} f(s) d s+\frac{1}{2} B \int_{x}^{1} e^{-(s-x) B} f(s) d s
\end{aligned}
$$

Which is equal

$$
\begin{aligned}
A u(x)= & +(I-Z)^{-1}\left(I-e^{-B}\right)^{-2}\left(A u_{0}-f(0)\right) e^{-x B}-(I-Z)^{-1}\left(I-e^{-B}\right)^{-2}\left(A u_{0}-f(0)\right) e^{-(1-x) B} \\
& -(I-Z)^{-1}\left(I-e^{-B}\right)^{-2}\left(A u_{0}+f(1)\right) e^{-(4-x) B}-(I-Z)^{-1}\left(I-e^{-B}\right)^{-2} e^{-(2+x) B} A u_{0} \\
& +(I-Z)^{-1}\left(I-e^{-B}\right)^{-2}\left(A u_{0}-f(0)\right) e^{-(3+x) B}-(I-Z)^{-1}\left(I-e^{-B}\right)^{-2} e^{-(1+x) B} A u_{0} \\
& +(I-Z)^{-1}\left(I-e^{-B}\right)^{-2}\left(A u_{0}-f(0)\right) e^{-(2-x) B}+(I-Z)^{-1}\left(I-e^{-B}\right)^{-2} e^{-(3-x) B} A u_{0} \\
& -\frac{1}{2}(I-Z)^{-1}\left(I-e^{-B}\right)^{-2}\left(e^{-x B} B \int_{0}^{1} e^{-s B}(f(s)-f(0)) d s-B \int_{0}^{1} e^{-(2+x+s) B} f(s) d s\right) \\
& -\frac{1}{2}(I-Z)^{-1}\left(I-e^{-B}\right)^{-2}\left(B \int_{0}^{1} e^{-(2+x-s) B} f(s) d s-B \int_{0}^{1} e^{-(4+x-s) B} f(s) d s\right) \\
& -(I-Z)^{-1}\left(I-e^{-B}\right)^{-2}\left(B \int_{0}^{1} e^{-(3-x+s) B} f(s) d s-e^{-(1-x) B} B \int_{0}^{1} e^{-s B}(f(s)-f(0)) d s\right) \\
& -\frac{1}{2}(I-Z)^{-1}\left(I-e^{-B}\right)^{-2}\left(B \int_{0}^{1} e^{-(2-x+s) B} f(s) d s-B \int_{0}^{1} e^{-(4-x+s) B} f(s) d s\right) \\
& -\frac{1}{2}(I-Z)^{-1}\left(I-e^{-B}\right)^{-2} B\left(\int_{0}^{1} e^{-(4-x-s) B} f(s) d s-e^{-(1-x) B} \int_{0}^{1} e^{-(1-s) B}(f(s)-f(1)) d s\right) \\
& +\frac{1}{2}(I-Z)^{-1}\left(I-e^{-B}\right)^{-2} e^{-(2-x) B} f(1)+\frac{1}{2}(I-Z)^{-1}\left(I-e^{-B}\right)^{-2} e^{-(5-x) B} f(1) \\
& +\frac{1}{2}(I-Z)^{-1}\left(I-e^{-B}\right)^{-2} e^{-(4+x) B} f(0)+\frac{3}{2}(I-Z)^{-1}\left(I-e^{-B}\right)^{-2} e^{-(1+x) B} f(0) \\
& +\frac{1}{2} B \int_{0}^{x} e^{-(x-s) B}(f(s)-f(x)) d s+\frac{1}{2} B \int_{x}^{1} e^{-(s-x) B}(f(s)-f(x)) d s \\
& -\frac{1}{2}(f(x)-f(0)) e^{-x B}-\frac{1}{2}(f(x)-f(1)) e^{-(1-x) B} \\
& +f(x)
\end{aligned}
$$

and by the same raisons as $u^{\prime \prime}$, we prove that :

$$
A u \in C([0,1] ; X) \text { and } u^{\prime \prime}(x)+A u(x)=f(x)
$$


Conversely, we suppose that statement 1 holds, i.e $u \in C^{2}([0,1] ; X) \cap C([0,1] ; D(A))$ then

$$
u_{0}=u(0) \in D(A)
$$

and

$$
A u_{0}-f(0)=-u^{\prime \prime}(0) \in \overline{D(A)}
$$

Finally we obtain the following maximal regularity:

Theorem 4.2. Let $f \in C^{\alpha}([0,1] ; X), 0<\alpha<1$, and we suppose that (1.3) holds, then the following assertions are equivalent

(1) The unique solution u of problem (1.1)-(1.2) has the maximal regularity property

$$
u^{\prime \prime}, A u \in C^{\alpha}([0,1] ; X)
$$

$$
u_{0} \in D(A), A u_{0}-f(0) \in(D(A), X)_{1-\frac{\alpha}{2},+\infty}
$$

Proof. Assume that there exists a strict solution $u$ of problem (1.1)-(1.2) having the maximal regularity property. From the previous theorem we have

$$
u_{0} \in D(A)
$$

Furthermore, the first and second terms in formula (4.1) are in $C^{\alpha}([0,1] ; X)$ and then

$$
\begin{aligned}
& e^{-B \cdot}\left(A u_{0}-f(0)\right) \in C^{\alpha}([0,1] ; X) \\
& e^{(1-\cdot) B}\left(A u_{0}-f(0)\right) \in C^{\alpha}([0,1] ; X)
\end{aligned}
$$

using remark, (f), page 39. in [14], we obtain

$$
A u_{0}-f(0) \in(D(B), X)_{1-\alpha, \infty}
$$

we recall that :

$$
(D(B), X)_{1-\alpha, \infty}=(D(A), X)_{1-\frac{\alpha}{2}, \infty}
$$

Conversely, assume that

$$
u_{0} \in D(A), A u_{0}-f(0) \in(D(A), X)_{1-\frac{\alpha}{2},+\infty}
$$


Using theorem, 1.4. Page 361 in [4] we have

$$
\begin{aligned}
& e^{-\sqrt{-A} \cdot}\left(A u_{0}-f(0)\right) \in C^{\alpha}([0,1] ; X) \\
& e^{(1-\cdot) \sqrt{-A}}\left(A u_{0}-f(0)\right) \in C^{\alpha}([0,1] ; X) \\
& \int_{0}^{1} B e^{-s B}(f(s)-f(0)) d s \in C^{\alpha}([0,1] ; X) \\
& \int_{0}^{1} B e^{-(1-s) B}(f(s)-f(1)) d s \in C^{\alpha}([0,1] ; X)
\end{aligned}
$$

and thus

$$
u^{\prime \prime}, A u \in C^{\alpha}([0,1] ; X)
$$

\section{Concrete application}

Let $X=L^{p}(\mathbb{R})$ and $f \in C^{\alpha}\left([0,1], L^{2}(\mathbb{R})\right), 0<\alpha<1$ and $1<p<\infty$. Consider the problem :

$$
\left\{\begin{array}{l}
\frac{\partial^{2} u}{\partial x^{2}}(x, y)+\frac{\partial^{2} u}{\partial y^{2}}(x, y)=f(x, y) \quad(x, y) \in[0,1] \times \mathbb{R} \\
u(0, y)=u_{0}(y), y \in \mathbb{R} \\
\frac{\partial u}{\partial x}(0, y)=\frac{\partial u}{\partial y}(1, y)
\end{array}\right.
$$

We dfine operator $A$ as follows

$$
\left\{\begin{array}{l}
D(A)=H^{2}(\mathbb{R}) \\
A u=u^{\prime \prime}
\end{array}\right.
$$

As $X$ is a Hilbert space, $D(A)$ is dense in $X$, moreover $(-A)$ is a self adjoint operator, then

$$
D(\sqrt{-A})=(D(A), X)_{\frac{1}{2}, 2}=\left(H^{2}(\mathbb{R}), L^{2}(\mathbb{R})\right)_{\frac{1}{2}, 2}=H^{1}(\mathbb{R})
$$

see [11]. Using Fourier transformation, we prove that $A$ verifies (1.3). The following result is a consequence of theorem 4.2

Proposition 5.1. Problem (5.1) has a unique strict solution u such that

$$
u \in C^{2}\left([0,1] ; L^{2}(\mathbb{R})\right) \cap C\left([0,1] ; H^{2}(\mathbb{R})\right)
$$

satisfying

$$
\begin{gathered}
u^{\prime \prime} \in C^{\alpha}\left([0,1] ; L^{2}(\mathbb{R})\right) \\
\text { if and only if }
\end{gathered}
$$




$$
u_{0} \in H^{2}(\mathbb{R}) \text { and } A u_{0}-f(0) \in\left(H^{2}(\mathbb{R}), L^{2}(\mathbb{R})\right)_{1-\frac{\alpha}{2}, \infty}=B_{2, \infty}^{\alpha}(\mathbb{R})
$$

(the Besov space $B_{2, \infty}^{\alpha}(\mathbb{R})$ is completely deseribed in Grisvard [7] )

Acknowledgments. The authors wish to warmly thank the referees for all their useful suggestions.

\section{ReFERENCES}

[1] W. Arendt, R. Chill, S. Fornaro and C.Poupaud., $L^{p}$ - maximal regularity for non-autonomous evolution equations, J. Differ. Equations, 237 (2007), 1-26.

[2] A. V. Balakrishnan., Fractional powers of closed operators and the semigroups generated by them, Pac. J. Math. 10 (1960), 419-437.

[3] C. J. K. Batty, R. Chill, S. Srivastava., Maximal regularity in interpolation spaces for second-order Cauchy problems, Oper. Theory: Adv. Appl. 250 (2015), 49-66.

[4] G. Da Prato., Abstract Differential Equations, Maximal Regularity, and Linearization, Proc. Symp. Pure Math. 45 (1986), Part 1.

[5] G. Da Prato and P.Grisvard., Sommes d'opérateurs linéaires et équations différentielles opérationnelles. J. Math. Pures Appl. IX Ser. 54 (1975), 305-387.

[6] A. Favini, R. Labbas, S. Maingot, H. Tanabe and A. Yagi., On the Solvability and the Maximal Regularity of Complete Abstract Differential Equations of Elliptic Type, Funkcial Ekvacioj, 47 (2004), 423-452.

[7] P. Grisvard., Spazi di Tracce e Applicazioni, Rend. Mat. (4), vol. 5, série VI (1972), 657-729.

[8] M. Haase., The Functional Calculus for Sectorial Operators, Oper. Theory Adv. Appl., vol. 69, Birkhuser-Verlag, Basel (2006).

[9] S. G. Krein., Linear differental equations in Banach space, Moscou, 1967; English Translation: AMS, Providence, (1971).

[10] J.L. Lions., Théorème de trace et d'interpolation I et II. Annali S.N.S.di Pisa, 13, (1959), 389-403 et 14, (1960), 317-331.

[11] J. L. Lions and J. Peetre., Sur une classe d'espaces d'interpolation, Inst. Hautes Etudes Sci. Publ. Math., 19 (1964), 5-68.

[12] A.Lunardi., Analytic Semigroups and Optimal Regularity in Parabolic Problems, Birkhâuser, Basel, (1995).

[13] C. Martinez and M. Sanz., Fractionnal powers of non densely defined operators. Annali della Scuola Normale Superiore di Pisa-Classe di scienze Ser. 4, 18 (1991). 443-454.

[14] E. Sinestrari., On the abstract Cauchy problem of parabolic type in space of continuous functions, J. Math. Anal. Appl. 66 (1985), 16-66.

[15] J. Tanabe., Equations of Evolution, Pitman, London, San Francisco, Melbourne, (1979).

[16] H. Triebel., Interpolation Theory,. Function Spaces, Differential Operators, Amsterdam, North Holland (1978). 